\title{
Efecto de las comunicaciones word of mouth y electronic word of mouth en las intenciones de recompra de una marca colombiana
}

\begin{abstract}
Resumen
El objetivo del presente artículo consiste en analizar el efecto de las comunicaciones informales offline $y$ online entre ellas mismas, y sobre las intenciones de recompra de la marca Totto por parte de los jóvenes universitarios. Para lograrlo, se utilizó una metodología cuantitativa cuya recolección de información se realizó a partir de la utilización de un cuestionario estructurado, aplicado a una muestra de 306 jóvenes de edades comprendidas entre los 16 y 24 años. Los resultados demuestran que los jóvenes son más propensos a repetir la compra cuando acuden a las recomendaciones o comentarios cuya fuente de información son los familiares, los amigos o los expertos, dejando a un lado las recomendaciones procedentes de las redes sociales o de Internet.
\end{abstract}

\author{
María Cristina Otero Gómez \\ Doctoranda en Marketing de la \\ Universidad de Valencia - España \\ Docente la Universidad de los Llanos \\ Villavicencio - Colombia \\ Correo electrónico \\ motero@unillanos.edu.co \\ 다이. orcid/0000-0002-3241-7877 \\ Google Scholar \\ Wilson Giraldo Pérez \\ Doctorando en Marketing de la \\ Universidad de Valencia - España \\ Docente de la Universidad de los \\ Llanos \\ Villavicencio - Colombia \\ Correo electrónico \\ wgiraldo@unillanos.edu.co \\ ำ orcid.org/0000-0001-8514-9805 \\ Google Scholar
}

Recibido: julio 03 de 2018

Aprobado: abril 14 de 2020

Palabras clave:

consumidor, intención de recompra, Electronic Word of Mouth, Word of mouth. 


\section{Effect of word of mouth and electronic word of mouth communications on the repurchase intentions of a Colombian brand}

\begin{abstract}
The objective of this article is to analyze the effect of offline and online informal communications, and on the intentions of young university students to repurchase the brand Totto. Quantitative methodology was used to achieve this objective, for which the collection of information was done using a structured questionnaire applied to a sample of 306 young people aged between 16 and 24 years. The results show that young people are more likely to repurchase when they heed recommendations or reviews from family members, friends or experts, leaving aside recommendations from social networks or the Internet.
\end{abstract}

Key words:

consumer, repurchase intention, Electronic Word of mouth, Word of Mouth. 


\section{Introducción}

Word of Mouth (WOM) es considerada una de las formas de comunicación con mayor influencia en el comportamiento de compra de los consumidores, puesto que ejerce un importante rol en la modificación de las actitudes y patrones de consumo (Brown \& Reingen, 1987; De Bruyn \& Lilien, 2008). La información transmitida a través de WOM permite a los consumidores identificar las marcas y los productos que mejor se ajustan a sus necesidades. Dicha información surge a partir de los comentarios basados en las experiencias y conocimientos de otros usuarios, por lo que puede considerarse una fuente más confiable en comparación con la publicidad tradicional. Para efectos del presente estudio se distinguen dos tipos de Word of Mouth: el WOM tradicional y el WOM electrónico (EWOM). El primero se refiere a la comunicación oral entre personas; el segundo corresponde a las declaraciones realizadas a través de Internet.

La población objeto de estudio corresponde a jóvenes universitarios colombianos quienes en algún momento han usado productos de la marca Totto. La elección de este segmento se sustenta en que mucho se ha escrito sobre la relación entre juventud y tecnología. En general, se caracteriza a los jóvenes como adictos a Internet, celulares y computadoras, creativos y entrenados en el uso de múltiples tecnologías (Lago, 2014, p.13). En esta línea, Duffett (2017) sostiene que el crecimiento fenomenal de la tecnología digital interactiva ha afectado a casi todos los aspectos de la vida cotidiana de los consumidores jóvenes en las últimas dos décadas (p.19), convirtiendo a este segmento en un reto para las empresas, puesto que cada vez son más difíciles de atraer y de retener. En tal sentido, Kietzmann, Hermkens, McCarthy y Silvestre (2011) señalan que los consumidores están cansados de la comunicación unidireccional, por el contrario, desean que las empresas los escuchen, se involucren de manera apropiada y les respondan" (p. 250). 
Lo anterior demuestra que para las empresas se convierte en foco de interés conocer los comentarios positivos y negativos que giran en torno a sus marcas y productos. Desde el punto de vista científico, se ha encontrado una cantidad significativa de investigaciones que consideran a WOM como uno de los factores más influyentes en el negocio (Moslehpour, Wong, Phammy \& Aulia, 2017). No obstante, para el caso colombiano se desconoce cuál es el resultado de esa influencia en el comportamiento del consumidor puesto que, por un lado, se encuentra la forma tradicional representada en la comunicación oral y, por otro lado, está el uso de las tecnologías de la información y las comunicaciones. Tsai, Kuo \& Tan (2017) explican que la diferencia entre los tipos de Word of Mouth es cómo el mensaje se entrega a la persona y las herramientas utilizadas en el proceso de entrega del mensaje (p.182).

Siendo así, los interrogantes de este estudio giran en torno a ¿cuál es el lugar que ocupan las comunicaciones informales en los consumidores jóvenes?, ¿cuál tiene mayor incidencia en la intención de recompra de los productos de la marca Totto?, por último, ¿qué relación existe entre WOM y EWOM? De este modo, el objetivo del presente artículo consiste en analizar el efecto de las comunicaciones informales offline y online entre ellas mismas, y sobre las intenciones de recompra de la marca Totto por parte de los jóvenes universitarios.

La estructura de este artículo está organizada en las siguientes secciones. En la primera, se presenta la revisión de la literatura científica que aborda los conceptos de WOM, EWOM e intención de recompra (IR). También se describen las razones que motivaron la selección de la marca Totto. Además, se plantean las hipótesis cuyo contraste permite esclarecer algunas de las cuestiones pendientes de resolver en la literatura. En la segunda, se describe la metodología implementada. En la tercera, se presentan los resultados y la discusión del estudio empírico que dan lugar a las conclusiones y limitaciones, las cuales permiten identificar futuras líneas de investigación. 


\section{WOM versus EWOM}

El término WOM, es parte de las comunicaciones informales que realizan los consumidores de manera independiente a los intereses de una organización; se trata de opiniones basadas en su experiencia o conocimiento sobre un bien, un servicio, una marca o una empresa. En el idioma castellano también es conocida como "voz a voz", "boca-oreja", "boca a boca" o "recomendación personal", entre otras expresiones. Generalmente, surge entre personas que carecen de un marcado interés comercial por persuadir a otra persona para que use o abandone determinado producto o marca; por tanto, no hay un incentivo particular que conlleve a la generación de comentarios positivos o negativos. En síntesis, se define como una conversación informal mediante la que se expresan y difunden opiniones de productos y marcas (HarrisonWalker, 2001).

Mientras WOM inicialmente se refiere a la idea de una conversación oral entre consumidores, la difusión mundial de Internet ha permitido una forma menos personal pero más omnipresente del WOM, Ilamado Electronic Word of Mouth o EWOM (Jalilvand \& Samiei, 2012, p. 593). De acuerdo con lo anterior, EWOM se define como "cualquier declaración positiva o negativa hecha por un cliente potencial, actual o anterior acerca de un producto o empresa, la cual se hace disponible a una multitud de gente e instituciones a través de la Internet" (Hennig-Thurau, Gwinner, Walsh \& Gremier, 2004, p. 39). Entre las ventajas del modo electrónico de esta forma de comunicación, está el hecho de que Internet permite a los consumidores postear sus ideas, dar comentarios, revisar y evaluar productos en los blogs, salas de discusión, páginas de revisión, grupos de noticias y redes sociales (Vahdati \& Mousavi, 2016, p. 9); también porque se toma como una fuente valiosa de información, por tratarse de una herramienta de comunicación persuasiva que ayuda a los 
consumidores a reducir sus dudas para posteriormente tomar una decisión de compra con el mayor grado de certeza posible (Lim, 2015, p. 86).

Una de las características del WOM es la naturalidad con que aflora la comunicación, es decir, que no hay una presión o influencia comercial que motive el surgimiento de las opiniones o comentarios (Kozinets, Valck, De Wojnicki \& Wilner, 2010). Esta situación sugiere que se trata de una fuente más creíble en comparación con EWOM, puesto que la información proviene de familiares, amigos, conocidos o expertos; lo que disminuye el riesgo de persuasión por parte de la empresa al considerar que éstas pueden generar EWOM como una estrategia de marketing. No obstante, WOM presenta limitaciones, puesto que la comunicación oral se esfuma tan pronto se pronuncia (Stern, 1994). De manera contraria, en el entorno online las conversaciones de los consumidores quedan registradas y esto permite que el mensaje pueda ser leído no solo en el momento en el que se transmite, sino también en momentos posteriores (Marhuenda, 2016, p.17).

Otro aspecto que se destaca tiene que ver con los resultados de las comunicaciones informales; de manera indistinta - tanto WOM como EWOMpueden generar influencia según el contenido de las opiniones o comentarios difundidos entre emisores y receptores. Por ejemplo, cuando el mensaje transmitido por los usuarios es positivo, su contenido influye significativamente en la actitud hacia la marca y la intención de compra (Kudeshia \& Kumar, 2017 , p. 313). Igualmente, existen situaciones donde se presentan actitudes de los consumidores orientadas a difundir información negativa sobre la marca, lo que resalta aún más el lugar que ocupa EWOM en la gestión de los medios para la construcción del valor de marca (Chang, Hsieh \& Tseng, 2013, p. 488).

Bajo este entendido, existen investigaciones que respaldan la importancia de esta forma de comunicación, puesto que se ha demostrado que la información 
derivada de fuentes no comerciales tiende a ser más influyente en la formación de la opinión pública del consumidor, en comparación con las fuentes comerciales (Aguilar, San Martín \& Payo, 2014, p.18). Autores como Hung \& Li (2007) afirman que a través de WOM / EWOM se obtiene una gran cantidad y diversidad de información que los mensajes comerciales rara vez pueden transmitir; esta situación se presenta cuando los usuarios de una marca discuten desde múltiples perspectivas incluyendo el precio, la reputación, la eficacia y los problemas potenciales.

\section{Intención de recompra (IR)}

Es la disposición de cada individuo para realizar otras compras de la misma compañía, basándose en su experiencia previa (Hellier, Geursen, Carr \& Rickard, 2003). Teniendo en cuenta el uso de las tecnologías de información y comunicación, Yan \& Yu (2013) explican que IR es la probabilidad subjetiva de que una persona compre continuamente productos o servicios del proveedor o tienda electrónica en el futuro. IR es considerada por diversos autores como una manifestación de varios factores, entre los que se cuentan: la lealtad del cliente, la satisfacción, la utilidad percibida, los incentivos ofrecidos por las empresas (Bhattacherjee, 2001; Gruen, Osmonbekov \& Czaplewski, 2006), la experiencia de la compra y el hábito de compra (Liao, Palvia \& Lin, 2006; Lin \& Lekhawipat, 2014).

Aunque se ha mencionado que WOM representa ventajas para el consumidor, es necesario considerar su impacto en la empresa. Para el caso de la comunicación oral y su relación con IR, se ha demostrado que cuando se presentan demasiados comentarios o evaluaciones positivas se reduce la confianza, en comparación con la situación en la que los usuarios reciben la cantidad adecuada de WOM positivo (Nghia, Thanh \& Bao, 2017, p. 121). Esta 
situación de desconfianza puede reducir el volumen de ventas, por lo que se afectan los intereses de la empresa.

Por su parte, los estudios que relacionan EWOM con IR destacan que en el entorno online los usuarios están expuestos a un gran volumen de información que en oportunidades no están en condiciones de procesar; por lo que estos individuos pueden generar rechazo hacia la plataforma y una desmotivación hacia el proceso de compra (Turetken \& Sharda, 2004; Sicilia \& Ruiz, 2010). En contraposición, también se encontró evidencia que apoya la relación positiva entre estas variables. Así pues, Vallejo, Redondo \& Acerete (2015, p. 71), infieren que la publicación de recomendaciones online que reúnan los requisitos de credibilidad, calidad y volumen adecuado influirán en última instancia en la intención de recompra online.

\section{Selección de la marca objeto de análisis}

Las razones que motivaron la selección de Totto se fundamentan en las variaciones que ha presentado la marca en los últimos años. Según la consultora Raddar, en 2014 Totto mostró un gran desempeño al estudiar el concepto del "precio de reserva", indicador del branding que mide cuántas veces un consumidor está dispuesto a pagar el precio mínimo de un producto; en esta oportunidad el estudio reveló que los consumidores estarían dispuestos a pagar hasta 35 veces por Totto (Expertos en marca, 2015). Luego, la revista Semana (2016) en su artículo "Estas son las marcas más queridas por los colombianos" destaca que entre las más apreciadas por los consumidores nacionales se encuentra Totto. Por su parte, en el Ministerio de Comercio, Industria y Turismo (MINCIT, 2017), destaca el posicionamiento de la marca en otros países y la considera un ejemplo en la dinamización de: la inversión extranjera directa, las exportaciones y la expansión internacional. Esto se debe a que en la actualidad la marca hace presencia en más de 57 países y posee alrededor de 
600 tiendas en todo el mundo (Totto, 2017). Además, la Federación Nacional de Comerciantes (Fenalco) y la Cámara Colombiana de Comercio Electrónico (CCCE) señalan que Totto es una de las marcas de mayor crecimiento y rápido desarrollo en cuanto al manejo y desempeño de sus plataformas virtuales (Cámara de Comercio de Bogotá, 2018). A partir de estas consideraciones, Totto es la marca seleccionada para analizar las variables del presente estudio.

Por lo anteriormente expuesto, esta investigación pretende reducir la brecha entre la teoría y la práctica con respecto a las relaciones y el efecto de las comunicaciones informales, para ello se plantearon las siguientes hipótesis:

H1. Existe una relación directamente proporcional entre las variables WOM y EWOM.

H2. Las comunicaciones EWOM que realizan los consumidores sobre una marca tienen relación directa con IR.

H3. Las comunicaciones WOM que realizan los consumidores sobre una marca tienen relación directa con IR.

Las hipótesis anteriores, permiten plantear el siguiente modelo de contraste: 


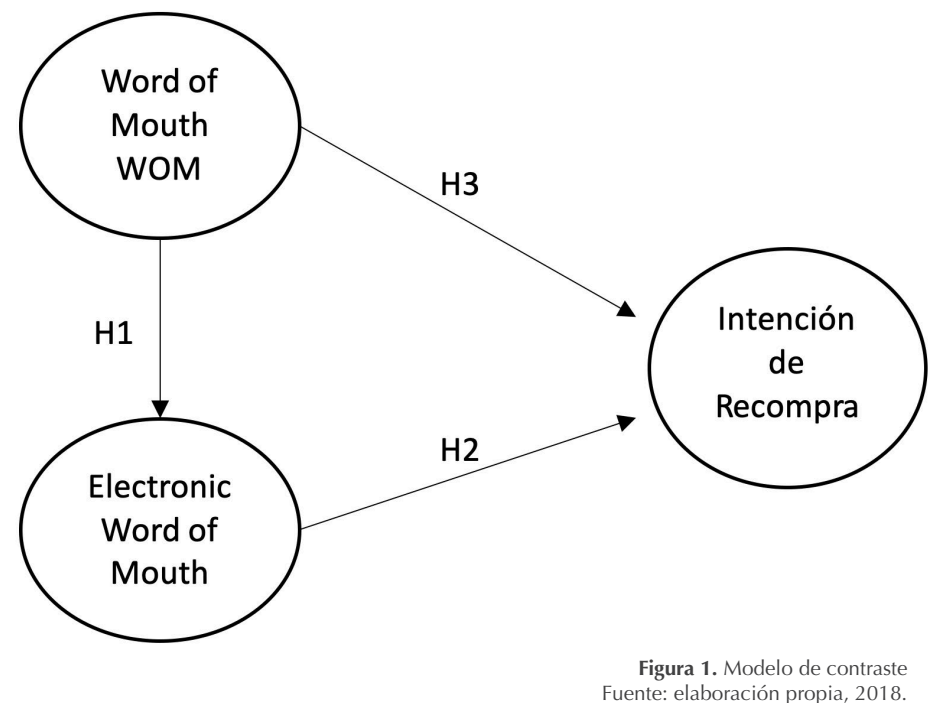

\section{Metodología}

Para la ejecución de la investigación se utilizó la metodología cuantitativa. El público objetivo son jóvenes universitarios entre 16 y 24 años, residentes en Colombia, compradores de productos de la marca Totto. Se aplicaron 321 cuestionarios, de los cuales se descartaron 15 por encontrarse errores en su diligenciamiento, para así finalizar con un tamaño de muestra de 306 individuos. Para alcanzar el objetivo de la investigación y contrastar las hipótesis planteadas, se diseñó un cuestionario estructurado con el fin de recopilar los datos primarios mediante entrevista personal. El procedimiento de selección de la muestra fue por conveniencia. La tabla 1 describe el perfil de la muestra, donde se observa que el mayor porcentaje de entrevistados son mujeres. Un alto porcentaje de los jóvenes pertenece al rango ubicado entre 
los 18 y 22 años (75.2\%); seguido de los menores de edad pertenecientes al segmento de 16 y 17 años (14.4\%); finalmente, quienes están entre los 23 y 24 años (10.4\%).

Tabla 1. Descripción de la muestra

\begin{tabular}{lccc}
\hline Género & $\begin{array}{c}\text { \% } \\
(\mathbf{n = 3 0 6 )}\end{array}$ & Edad & $\begin{array}{c}\text { \% } \\
(\mathbf{n = 3 0 6})\end{array}$ \\
\hline Femenino & $54,2 \%$ & 16 años & $2,6 \%$ \\
Masculino & $45,8 \%$ & 17 años & $11,8 \%$ \\
Total & $100 \%$ & 18 años & $14,7 \%$ \\
\hline Estratificación socioeconómica & $\%$ & 20 años & $21,9 \%$ \\
& $(\mathbf{n = 3 0 6 )}$ & 21 años & $17,3 \%$ \\
Estrato 1 y 2 & $46,1 \%$ & 22 años & $10,8 \%$ \\
Estrato 3 y 4 & $52,6 \%$ & 23 años & $10,5 \%$ \\
Estrato 5 & $1,3 \%$ & 24 años & Total \\
Total & $100 \%$ & $4,6 \%$ & $100 \%$ \\
\hline
\end{tabular}

Fuente: elaboración propia, 2018.

\section{Medición de las variables}

La medición del concepto WOM se realizó a través de 4 ítems a partir del trabajo de Carroll \& Ahuvia (2006). Adicionalmente, EWOM está compuesto por 3 ítems adaptados del trabajo de Karjaluoto, Munnukka \& Kiuru (2016). Para medir IR se utilizaron 3 ítems a partir de los trabajos de Wang, Yeh \& Liao (2013); Alavi, Rezaei, Valaei \& Wan (2016). Por último, todas las variables se consideraron como constructos unidimensionales para realizar su medición.

En todos los casos los ítems fueron medidos mediante una escala Likert de 5 puntos desde (1) "Totalmente en desacuerdo" a (5) "Totalmente de acuerdo". En el anexo 1 se describe cómo se realizó la medición de las variables utilizadas en la presente investigación. 
Para evaluar la calidad del instrumento de medida y plantear el modelo estructural se utilizó la técnica de regresión por mínimos cuadrados parciales (PLS), también se utilizó el software SPSS para calcular las correlaciones Tau-c de Kendall y de esta forma comprobar las hipótesis.

\section{Modelo de medida}

Para corroborar la validez, la fiabilidad y la consistencia del instrumento, se realizó una evaluación del modelo de medida, tal como se muestra en las tablas 2 y 3 . Los resultados demuestran el cumplimiento de los valores para las cargas según la propuesta de Bagozzi \& Yi (1988) quienes proponen que una carga factorial debe ser superior a 0,6. Para la fiabilidad compuesta, se atendió la recomendación de Chin (1998) y de Steenkamp \& Van Trijp (1991) considerándose apropiados los índices superiores a 0,7. Para el AVE, se comprobó que todos los valores cumplieran con la norma recomendada de 0,50, según los postulados de Fornell \& Larcker (1981). Para el Alfa de Cronbach se revisó que el valor fuera superior a 0,7 (Nunnally, 1978). Para la validez discriminante se utilizó el criterio de cargas cruzadas en el cual ningún ítem debe cargar más alto en otro constructo en relación con el propio constructo que se pretende medir en el modelo (Barclay, Thompson \& Higgins, 1995). De este modo, se evidencia que existe adecuada consistencia interna, fiabilidad y validez convergente y discriminante, razón por la cual se procede a la comprobación de las hipótesis planteadas. 
Otero-Gómez, M. C. y Giraldo-Pérez, W. / Efecto de las comunicaciones word of mouth y electronic word of mouth en las intenciones de recompra de una marca colombiana

Tabla 2. Evaluación del modelo de medida

\begin{tabular}{|c|c|c|c|c|c|c|c|}
\hline \multicolumn{2}{|c|}{ Ítems } & \multirow{2}{*}{$\begin{array}{c}\text { Cargas } \\
0,829\end{array}$} & \multirow{2}{*}{$\begin{array}{c}\text { T Value } \\
45,709\end{array}$} & \multirow{2}{*}{$\begin{array}{c}\text { P Value } \\
0,000\end{array}$} & \multirow[t]{2}{*}{ FC } & \multirow[t]{2}{*}{ AVE } & \multirow[t]{2}{*}{ ALFA } \\
\hline \multirow{4}{*}{ WOM } & WOM1 & & & & & & \\
\hline & WOM2 & 0,823 & 41,084 & 0,000 & \multirow{3}{*}{0,897} & \multirow{3}{*}{0,686} & \multirow{3}{*}{0,847} \\
\hline & WOM3 & 0,853 & 49,678 & 0,000 & & & \\
\hline & WOM4 & 0,807 & 25,597 & 0,000 & & & \\
\hline \multirow{3}{*}{ EWOM } & EWOM1 & 0,894 & 58,315 & 0,000 & \multirow{3}{*}{0,917} & \multirow{3}{*}{0,786} & \multirow{3}{*}{0,863} \\
\hline & EWOM2 & 0,898 & 50,635 & 0,000 & & & \\
\hline & EWOM3 & 0,867 & 39,824 & 0,000 & & & \\
\hline \multirow{3}{*}{ IR } & IR1 & 0.931 & 96,55 & 0,000 & \multirow{3}{*}{0,941} & \multirow{3}{*}{0,842} & \multirow{3}{*}{0,906} \\
\hline & IR2 & 0.927 & 84,127 & 0,000 & & & \\
\hline & IR3 & 0.895 & 67,296 & 0,000 & & & \\
\hline
\end{tabular}

Fuente: elaboración propia, 2018.

Tabla 3. Validez discriminante cargas cruzadas ítem/constructo

\begin{tabular}{|c|c|c|c|}
\hline Ítem / constructo & WOM & EWOM & IR \\
\hline WOM1 & 0,829 & 0,398 & 0,587 \\
\hline WOM2 & 0,823 & 0,588 & 0,526 \\
\hline WOM3 & 0,853 & 0,498 & 0,539 \\
\hline WOM4 & 0,807 & 0,448 & 0,505 \\
\hline EWOM1 & 0,520 & 0,894 & 0,362 \\
\hline EWOM2 & 0,537 & 0,898 & 0,406 \\
\hline EWOM3 & 0,487 & 0,867 & 0,428 \\
\hline IR1 & 0,601 & 0,407 & 0,931 \\
\hline IR2 & 0,612 & 0,379 & 0,927 \\
\hline IR3 & 0,588 & 0,454 & 0,895 \\
\hline
\end{tabular}

Fuente: elaboración propia, 2018. 


\section{Resultados}

La bondad predictiva del constructo dependiente quedó demostrada mediante el cálculo de la varianza explicada (R2) cuyo valor de 0,452 evidencia un efecto mediano pero relevante en la intención de recompra juvenil.

Los resultados expuestos en la tabla 4 y en la figura 2 corresponden a la muestra total de jóvenes, en la cual una vez revisados los valores de coeficientes path $(\beta)$ y de correlaciones Tau-c Kendall ( $\tau$ ) se evidencia que WOM tiene una relación significativa y directamente proporcional sobre EWOM, por tanto, se acepta $\mathrm{H} 1$. De otro lado, los resultados demuestran que las comunicaciones WOM que sostienen los consumidores en relación con una marca, tienen una relación directa y significativa sobre IR, en este sentido se acepta $\mathrm{H} 3$. Finalmente, no es posible aceptar $\mathrm{H} 2$ puesto que, a pesar de ser significativa la relación entre EWOM e IR, el valor del coeficiente path es menor al valor de 0,2 propuesto por Chin (1998).

Tabla 4. Comprobación de hipótesis

\begin{tabular}{ccccc}
\hline Hipótesis & \multicolumn{2}{c}{ Coeficiente $\boldsymbol{\beta}$} & t value & p value \\
\hline H1 WOM -> EWOM & 0,581 & $* * * *$ & 12,446 & 0,000 \\
\hline H2 EWOM -> IR & 0,107 & $* *$ & 2,021 & 0,044 \\
\hline H3 WOM -> IR & 0,593 & $* * * *$ & 11,190 & 0,000 \\
\hline Hipótesis & Coeficiente $\boldsymbol{T}$ & t value & p value \\
\hline H1 WOM -> EWOM & 0,421 & ${ }^{* * * *}$ & 12,040 & 0,000 \\
\hline H2 EWOM -> IR & 0,308 & $* * *$ & 5,635 & 0,012 \\
\hline H3 WOM -> IR & 0,497 & $* * * *$ & 15,220 & 0,000 \\
\hline
\end{tabular}

Nota: n. s: no significativo; ${ }^{* * * *}$ Valor $\mathrm{t}>3,310(\mathrm{p}<0,001) ;{ }^{* * *}$ Valor $\mathrm{t}>2,586(\mathrm{p}<0,01) ;{ }^{* *}$ Valor $\mathrm{t}>1,965(\mathrm{p}<0,05) ;{ }^{*}$ Valor $\mathrm{t}>1,645(\mathrm{p}<0,10)$. Fuente: elaboración propia, 2018. 
En la figura 2 se demuestra el cumplimiento de dos de las tres hipótesis planteadas en el modelo de teórico.

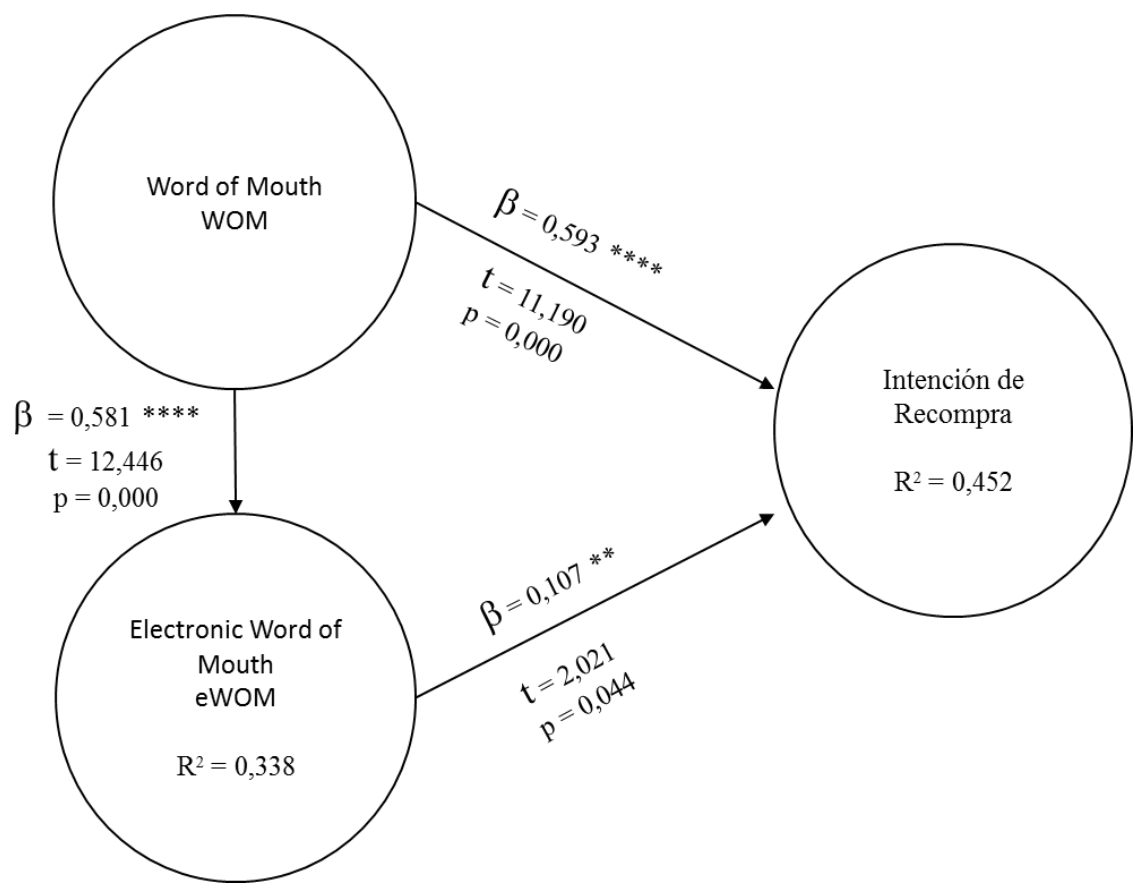

Figura 2. Estimación del Modelo Estructural.

Fuente: elaboración propia, 2018.

Los resultados permiten afirmar que es alta la intensidad que existe en la relación entre las comunicaciones WOM que sostienen los jóvenes y su intención de recompra $(\beta=0,593 ; \tau=0,497)$. 


\section{Discusión teórica}

La presente investigación contribuye a la literatura en el ámbito de las comunicaciones informales y su influencia en el comportamiento de los consumidores jóvenes universitarios. En ella se introdujo un modelo conceptual que demostró la relación existente entre las diferentes formas de WOM online y offline y sus efectos en IR.

En primer lugar, se exploró la existencia de una relación directamente proporcional entre WOM y EWOM $(\mathrm{H} 1)$. Coherentemente con investigaciones previas, en las que se evidencia que EWOM es subsecuente de WOM (Romero \& Ruiz, 2020), este estudio demuestra que es muy probable que un consumidor antes de realizar opiniones y comentarios sobre la marca en los entornos virtuales, se incline por compartir información a través de WOM tradicional cuyo contacto inicial son sus grupos de referencia y de filiación.

En segundo lugar, se planteó la existencia de una relación directa entre EWOM e IR (H2). En este sentido, los resultados de la presente investigación rechazan la hipótesis, aproximándose a las aportaciones de Matute, Polo \& Utrillas (2016), quienes en su estudio demostraron que no existe un efecto directo entre estas variables, esto se atribuye a que aspectos como la cantidad y la credibilidad de la información generan desconfianza en el consumidor. Este análisis lo refuerzan Nghia et al. (2017) al afirmar que cuando se presentan demasiados comentarios positivos se reduce la confianza, lo que conlleva a la disminución de IR.

Por último, los hallazgos frente a la tercera hipótesis, relacionada con el efecto de WOM sobre IR, concuerdan con lo planteado por Moslehpour et al. (2017), al considerar el WOM como uno de los factores más influyentes en un negocio; un coeficiente de correlación cercano a 0,5 indica que a medida que 
aumentan los comentarios informales con referencia a la marca Totto, también lo hace la intención que tienen los jóvenes de realizar procesos de recompra de dicha marca. Este resultado también guarda relación con la investigación de Meuter, McCabe \& Curran (2013), quienes sugieren la conveniencia de alentar a un consumidor a participar en WOM, puesto que parece ser mucho más poderoso que persuadirlo a publicar un comentario favorable a través de las diferentes formas de comunicación EWOM.

De acuerdo con lo anterior, es importante comprender el tipo de relación que existe entre el emisor y el receptor o receptores, toda vez que el conocimiento de la fuente de información es lo que maximiza la intensidad del efecto en el comportamiento de los consumidores. Esto se debe a que los individuos generalmente confían más en las opiniones y comentarios de las personas conocidas, tales como la familia o los amigos cercanos, frente a la comunicación emitida mediante el ejercicio publicitario, por lo que en esta relación el WOM es la variable más influyente sobre IR. Este hallazgo contribuye a profundizar la investigación previa que hasta ahora ha proporcionado pruebas limitadas sobre los efectos de la relación mencionada.

\section{Implicaciones prácticas}

A partir de los resultados planteados en párrafos anteriores, esta investigación ofrece 2 contribuciones prácticas enfocadas al mundo de las comunicaciones empresariales. La primera, está relacionada con el mensaje que la marca debe incorporar en sus comunicaciones. De esta forma el gerente de mercadeo o encargado del área de la comunicación de marca, debe reforzar los lazos y la cercanía de los influenciadores directos del usuario del producto en las diferentes piezas publicitarias utilizadas en las campañas. En estos casos se debe resaltar la notoriedad de la marca Totto demostrando su funcionalidad en las situaciones cotidianas del segmento joven. 
Una segunda contribución consiste en recomendar para el diseño de las diferentes piezas gráficas o audiovisuales, que se privilegie el uso de imágenes relativas a la amistad o a las relaciones afectivas, por encima de aquellas que refieran las relaciones familiares. Lo anterior se debe a que en la edad que tiene el grupo objetivo estudiado, los comentarios de su grupo social de referencia tienen mayor presión e implicación, en comparación con los comentarios provenientes de sus grupos de filiación.

\section{Conclusiones, limitaciones y futuras líneas de investigación}

Muchas organizaciones y sus marcas han utilizado las comunicaciones de marketing en redes sociales sin comprender realmente los efectos que estos canales digitales de comunicación ejercen en las respuestas actitudinales de los consumidores jóvenes (Duffett, 2017, p. 32). Para el caso de la marca Totto, los resultados obtenidos con el desarrollo del presente estudio demuestran y permiten concluir que este consumidor encuentra mayor credibilidad para su decisión de recompra en WOM en comparación con EWOM, pese a que la literatura refiere que los jóvenes en la actualidad consumen tecnología excesivamente (Upegui, 2017, p. 53). Totto cuenta con una página Web que permite realizar compras en línea, así como plataformas de redes sociales entre las que se cuentan Facebook, Instagram, Twitter y YouTube; sin embargo, pareciera ser que los consumidores no perciben estas herramientas como fuentes lo suficientemente creíbles o convincentes para repetir sus compras.

Pese a que esta investigación ha contribuido en reducir la brecha académicoprofesional en materia de las comunicaciones sobre productos y marcas, presenta las siguientes limitaciones: 1). Se trata de un estudio realizado en un periodo corto, lo que impide representar la dinámica propia de una investigación longitudinal. 2). Las preguntas del cuestionario involucraron todas las herramientas del entorno online, por tanto, el hallazgo es incapaz 
de explicar el efecto por separado de cada una de las redes sociales. 3). Las respuestas corresponden a jóvenes pertenecientes a los estratos medio y bajo de la escala social, por lo que no podría generalizarse al comportamiento de todos los jóvenes.

Por lo anterior, esta investigación también considera importante realizar futuros estudios orientados a comprender aspectos relacionados con el uso de las redes sociales y la actitud de los consumidores jóvenes hacia las comunicaciones de marketing en estos canales. Adicionalmente, resulta de especial interés profundizar en la percepción que tienen los jóvenes en cuanto a la credibilidad, la calidad y la cantidad de información comercial que proporciona Internet.

\section{Apoyos y agradecimientos}

Los autores agradecen al Departament de Comercialització i Investigació de Mercats de la Universitat de València (España) y a la Dirección General de Investigaciones de la Universidad de los Llanos (Colombia), por respaldar la investigación "Antecedentes y consecuencias del valor de marca. Un estudio centrado en los consumidores jóvenes colombianos", de la cual se desprende este artículo. También agradecemos el apoyo recibido del programa "Colombia Científica y su componente Pasaporte a la Ciencia", en el marco del Foco Sociedad - Reto 2: Innovación social para el desarrollo económico y la inclusión productiva. 


\section{Referencias}

Aguilar, V., San Martin, S. \& Payo, R. (2014). La aplicación empresarial del marketing viral y el efecto boca-oreja electrónico. Opiniones de las empresas. Cuadernos de Gestión, 14(1), 15-31. https://www.redalyc.org/ pdf/2743/274330593001.pdf

Alavi, S. A., Rezaei, S., Valaei, N. \& Wan, W. K. (2016). Examining shopping mall consumer decision-making styles, satisfaction and purchase intention. The International Review of Retail, Distribution and Consumer Research, 26(3), 272 -303. http://dx.doi.org/10.1080/09593969.2015.1096808

Bagozzi, P. R. \& Yi, Y. (1988). On the evaluation of structural equation model. Journal of the academy of marketing science, 16(1), 74-94. https://doi. org/10.1007/BF02723327

Barclay, D., Thompson, R. \& Higgins, C. (1995). The partial least squares (PLS) approach to causal modelling: personal computer adoption and use as illustration. Technology Studies, 2(2), 285-309. https://www.researchgate. net/publication/242663837_The_Partial_Least_Squares_PLS_Approach_ to_Causal_Modeling_Personal_Computer_Use_as_an_Illustration

Bhattacherjee, A. (2001). An empirical analysis of the antecedents of electronic commerce service continuance. Decision Support Systems, 32, 201-214. https://doi.org/10.1016/S0167-9236(01)00111-7

Brown, J. J. \& Reingen, P. H. (1987). Social ties and word-of-mouth referral behavior. Journal of Consumer Research, 14(3), 350-362. http://www.jstor. org/stable/2489496 
Cámara de Comercio de Bogotá. (2018). El comercio electrónico de moda en Colombia crece por encima del canal tradicional. https://www.ccb.org. co/Clusters/Cluster-de-Joyeria-y-Bisuteria/Noticias/2018/Febrero-2018/ El-comercio-electronico-de-moda-en-Colombia-crece-por-encima-delcanal-tradicional

Carroll, B. A. \& Ahuvia, A. C. (2006). Some antecedents and outcomes of brand love. Marketing Letters, 17(2), 79-89. https://doi.org/10.1007/s11002-0064219-2

Chang, A., Hsieh, S. H. \& Tseng, T. H. (2013). Online brand community response to negative brand events: the role of group Ewom. Internet Research, 23(4), 486-506. https://doi.org/10.1108/IntR-06-2012-0107

Chin, W. (1998). Issues and opinion on structural equation modeling. MIS Quarterly, 22(1), 7-17. http://www.jstor.com/stable/249674

De Bruyn, A. \& Lilien, G. L. (2008). A multi-stage model of word-of-mouth influence through viral marketing. International Journal of Research in Marketing 25, 151-163. https://doi.org/10.1016/j.ijresmar.2008.03.004

Duffett, R. G. (2017). Influence of social media marketing communications on young consumers' attitudes. Young Consumers, 18(1), 19-39. https://doi. org/10.1108/YC-07-2016-00622

Expertos en marca. (2015). Totto la marca que superó a Adidas. Expertos en Marca. http://www.expertosenmarca.com/totto-la-marca-que-supero-adidas/

Fornell, C. \& Larcker, D. F. (1981). Evaluating structural equations models with unobservable variables and measurement error. Journal of Marketing Research, 18, 39-50. https://www.jstor.org/stable/3151312 
Gruen, T. W., Osmonbekov, T. \& Czaplewski, A. J. (2006). EWOM: The impact of customer-to-customer online Know-How exchange on customer value and loyalty. Journal of Business Research, 59, 449-456. https://doi. org/10.1016/j.jbusres.2005.10.004

Harrison-Walker, L. J. (2001). The measurement of word-of-mouth communication and an investigation of service quality and customer commitment as potential antecedents. Journal of Service Research, 4, 60-75. https://doi.org/10.1177/109467050141006

Hellier, P., Geursen, G., Carr, R. \& Rickard, J. (2003). Customer repurchase intention:Ageneral structural equation model. European JournalofMarketing, 37(11/12), 1762-1800. http://dx.doi.org/10.1108/03090560310495456

Hennig-Thurau, T., Gwinner, K. P., Walsh, G. \& Gremier, D. D. (2004). Electronic word-of-mouth via consumer-opinion platforms: what motivates consumers to articulate themselves on the Internet? Journal of interactive marketing, 18(1), 38-52. https://search.proquest.com/docview/229636468 ?accountid $=14777$

Hung, K. H. \& Li, S. T. (2007). The influence of eWOM on virtual consumer communities: social capital, consumer learning, and behavioral outcomes. Journal of Advertising Research, 47(4), 485-495. http://www. journalofadvertisingresearch.com/content/47/4/485

Jalilvand, M. R. \& Samiei, N. (2012). The impact of electronic word of mouth on a tourism destination choice. Internet Research, 22(5), 591-612. http://dx.doi.org/10.1108/10662241211271563

Karjaluoto, H., Munnukka, J. \& Kiuru, K. (2016). Brand love and positive word of mouth: the moderating effects of experience and price. Journal of Product \& Brand Management, 25(6), 527 - 537. http://dx.doi.org/10.1108/ JPBM-03-2015-0834 
Kietzmann, J. H., Hermkens, K., McCarthy, I. P. y Silvestre, B. S. (2011). Social media? Get serious! Understanding the functional building blocks of social media. Business Horizons 54(3), 241-251. https://doi.org/10.1016/j. bushor.2011.01.005

Kozinets, R. V, Valck, K. De Wojnicki, A. C. \& Wilner, S. J. (2010). Networked narratives: understanding word-of-mouth marketing in online communities. Journal of Marketing, 74(2), 71-89. https://doi.org/10.1509/jm.74.2.71

Kudeshia, Ch. \& Kumar, A. (2017). Social eWOM: does it affect the brand attitude and purchase intention of brands? Management Research Review, 3, 310 - 330. https://doi.org/10.1108/MRR-07-2015-0161

Lago, M. S. (2014). Los jóvenes, las tecnologías y la escuela. Revista Integra Educativa, 7(3), 5-20. http://www.scielo.org.bo/scielo.php?pid=S199740432014000300001\&script=sci_arttext

Liao, C., Palvia, P. \& Lin H. N. (2006). The roles of habit and web site quality in e-commerce. International Journal of Information Management, 26(6), 469-483. https://doi.org/10.1016/j.ijinfomgt.2006.09.001

Lim, M. W. (2015). The influence of internet advertising and electronic word of mouth on consumer perceptions and intention: some evidence from online group buying. The Journal of Computer Information Systems, 55(4), 81-89. https://doi.org/10.1080/08874417.2015.11645790

Lin, C. \& Lekhawipat, W. (2014). Factors affecting online repurchase intention. Industrial Management \& Data Systems, 114(4), 597-611. https://doi. org/10.1108/IMDS-10-2013-0432

Marhuenda, G. C. (2016). La influencia del Word of Mouth electrónico sobre las ventas de un refresco (Tesis doctoral). Universidad Complutense de Madrid, España. https://eprints.ucm.es/40633/ 
Matute, J., Polo, R. Y. \& Utrillas, A. (2016). The influence of EWOM characteristics on online repurchase intention. Online Information Review, 40(7), 1090-1110. https://doi.org/10.1108/OIR-11-2015-0373

Meuter, M. L., McCabe, D. B. \& Curran, J. M. (2013). Electronic word-ofmouth versus interpersonal word-of-mouth: are all forms of word-ofmouth equally influential? Services Marketing Quarterly, 34(3), 240-256. https://doi.org/10.1080/15332969.2013.798201

Ministerio de Comercio, Industria y Turismo (MINCIT). (2017). Informe al Congreso 2016-2017 Sector Comercio, Industria y Turismo. https://www. mincit.gov.co/CMSPages/GetFile.aspx?guid=fa764aa1-f6f4-4dc2-bc3f0050db08ab0e

Moslehpour, M., Wong, W., Phamy, K. V. \& Aulia, C. K. (2017). Repurchase intention of Korean beauty products among Taiwanese consumers. Asia Pacific Journal of Marketing and Logistics, 29(3), 569-588. https://doi. org/10.1108/APJML-06-2016-0106

Nghia, H. T., Thanh, B. T. \& Bao, D. Q. (2017). The relationship between satisfaction, trust and repurchase intention: examining the moderating role of word of mouth. Journal of Science Ho Chi Minh City Open University, 7(3), 111-124. http://journalofscience.ou.edu.vn/index.php/econ-en/article/ view/191

Nunnally, J. C. (1978). Psychometric Theory. New York: McGraw Hill.

Romero, J. \& Ruiz, E. D. (2020). Be a part of it: promoting WOM, eWOM, and content creation through customer identification. Spanish Journal of Marketing - ESIC, 24(1), 55-72. https://doi.org/10.1108/SJME-11-2019-0092

Semana (20 de junio de 2016). Estas son las marcas más queridas por los colombianos. Semana. http://www.semana.com/economia/articulo/lasmarcas-mas-queridas-por-los-colombianos/478530 
Sicilia, M. \& Ruiz, S. (2010). The effects of the amount of information on cognitive responses in online purchasing tasks. Electronic Commerce Research and Applications, 9, 183-191. https://doi.org/10.1016/j.elerap.2009.03.004

Steenkamp, J. B. \& Van Trijp, H. (1991). The use Lisrel in validating marketing constructs. International Journal of Research in Marketing, 8, 283-299. https://doi.org/10.1016/0167-8116(91)90027-5

Stern, B. B. (1994). A revised communication model for advertising: multiple dimensions of the source, the message, and the recipient. Journal of Advertising, 23(2), 5-15. https://doi.org/10.1080/00913367.1994. 10673438

Totto. (2017). Quiénes somos. https://www.totto.cl/quienes-somos

Tsai, Ch. D., Kuo, Ch. \& Tan, M. J. (2017). The antecedents and consequences of word of mouth: a meta-analysis. Journal of Social and Administrative Sciences, 4(2), 181- 192. http://kspjournals.org/index.php/JSAS/article/ view/1327/1314

Turetken, O. \& Sharda, R. (2004). Development of a Fisheye-based information search processing aid (FISPA) for managing information overload in the web environment. Decision Support Systems, 37(3), 415-434. https://doi. org/10.1016/S0167-9236(03)00047-2

Upegui, C. M. (2017). El consumo tecnológico de los jóvenes...un desafío para los docentes. Colección Académica de Ciencias Sociales, 4(1), 51-60. https://www.semanticscholar.org/paper/El-consumo-tecnol\%C3\%B3gicode-los-j\%C3\%B3venes \%E2\%80\%A6Un-desaf\%C3\%ADo-C\%C3\%B3rdo ba/9e2a10f305af16656a3862d7488ce540127b3881?p2df 
Vahdati, H. \& Mousavi, S. H. (2016). Brand personality toward customer purchase intention: The intermediate role of electronic word-of-mouth and brand equity. Asian Academy of Management Journal, 21(2), 1-26. http://dx.doi.org/10.21315/aamj2016.21.2.1

Vallejo, J. M., Redondo, P. Y. \& Acerete, A. U. (2015). Las características del boca-oído electrónico y su influencia en la intención de recompra online. Revista Europea de Dirección y Economía de la Empresa, 24(2), 61-75. https://doi.org/10.1016/j.redee.2015.03.002

Wang, Y. S., Yeh, CH. H. \& Liao, Y. W. (2013). What drives purchase intention in the context of online content services? The moderating role of ethical selfefficacy for online piracy. International Journal of Information Management 33(1), 199-208. https://doi.org/10.1016/j.ijinfomgt.2012.09.004

Yan, W. S. \& Yu, Ch. H. (2013). Factors of influencing repurchase intention on deal of the day group buying website. Hong Kong Baptist University: Hong Kong. https://www.semanticscholar.org/paper/Factors-of-influencing-repurchaseintention-on/5d71a67989a0570405ee5c244eddfa58d35eb56d

Como citar: Otero-Gómez, M. C. y Giraldo-Pérez, W. (2020). Efecto de las comunicaciones word of mouth y electronic word of mouth en las intenciones de recompra de una marca colombiana. Revista KEPES, 17(22), 229-255. https://doi.org/10.17151/kepes.2020.17.22.9 


\section{Anexo 1}

Escalas de medición

\begin{tabular}{|c|c|c|}
\hline Dimensión & Pregunta & Fuente \\
\hline \multirow{4}{*}{ WOM } & WOM1. Recomiendo la marca Totto a mucha gente & \multirow{4}{*}{$\begin{array}{l}\text { Carroll \& Ahuvia } \\
(2006)\end{array}$} \\
\hline & WOM2. Hablo de Totto a mis amigos & \\
\hline & WOM3. Trato de difundir buenas palabras sobre la marca Totto & \\
\hline & WOM4. Doy a la marca Totto muchos comentarios positivos & \\
\hline \multirow{3}{*}{ EWOM } & \multicolumn{2}{|l|}{ EWM1. Hablo de Totto en entornos online } \\
\hline & $\begin{array}{l}\text { EWM2. Le doy a la marca Totto muchos comentarios positivos en } \\
\text { Internet }\end{array}$ & $\begin{array}{l}\text { Karjaluoto, Munnukka } \\
\text { \& Kiuru (2016) }\end{array}$ \\
\hline & $\begin{array}{l}\text { EWM3. Trato de difundir buenos comentarios sobre Totto en } \\
\text { Internet }\end{array}$ & \\
\hline \multirow{3}{*}{ Intención de compra } & IRC1. Seguiré pagando por productos de la marca Totto & \multirow{3}{*}{$\begin{array}{l}\text { Wang, Yeh \& Liao } \\
\text { (2013); Alavi, Rezaei, } \\
\text { Valaei \& Wan (2016) }\end{array}$} \\
\hline & $\begin{array}{l}\text { IRC2. Tengo la intención de seguir comprando la marca Totto en } \\
\text { el futuro }\end{array}$ & \\
\hline & $\begin{array}{l}\text { IRC3. Seguiría comprando productos de la marca Totto en lugar } \\
\text { de cualquier otra marca disponible }\end{array}$ & \\
\hline
\end{tabular}

Fuente: elaboración propia (2018). 